\title{
Initial experience with dual-layer detector spectral CT for diagnosis of blood or contrast after endovascular treatment for ischemic stroke
}

\author{
Marie Louise E. Bernsen ${ }^{1}$ - Peter B. Veendrick ${ }^{1}$. Jasper M. Martens ${ }^{1}$ - Milan E. J. Pijl ${ }^{1}$. Jeannette Hofmeijer ${ }^{2,3}$. \\ Maarten J. van Gorp ${ }^{1}$
}

Received: 31 March 2021 / Accepted: 20 May 2021

(c) The Author(s), under exclusive licence to Springer-Verlag GmbH Germany, part of Springer Nature 2021

\begin{abstract}
Purpose To determine whether spectral detector CT (SDCT) with a plain non-enhanced monochromatic CT, a water-weighted image after iodine removal, an iodine map, and Mono energetic images changes the diagnosis and classification of intracranial hemorrhage based on single energy CT after endovascular treatment (EVT) for ischemic stroke.

Methods Two readers evaluated single energy and SD CT data collected from 63 patients within one week after EVT. They diagnosed ICH or contrast staining, and graded ICH according to the Heidelberg and Safe Implementation of Thrombolysis in Stroke-Monitoring Study (SITS-MOST) classification. Differences in diagnosis between single energy and SD CT were tested with Pearson's chi-squared test. Diagnostic values of single energy CT were calculated. Interrater agreement was based on Cohen's Kappa.

Results When spectral data were added to single energy CT, the diagnosis of ICH changed in 8 CT scans (13\%): in 4, the diagnosis of ICH was rejected and in 4, initially undetected ICH was diagnosed. In an additional 3 patients, the ICH grade was modified. CT alone had $88 \%$ sensitivity, $87 \%$ specificity, $88 \%$ positive diagnostic value, $87 \%$ negative diagnostic value, and $87 \%$ overall accuracy for ICH compared to SDCT. Interreader agreement on the presence of ICH was 0.84 (95\% CI 0.51-0.86) for spectral CT and 0.84 (95\% CI 0.73-0.97) for single energy CT.
\end{abstract}

Conclusion SD CT after endovascular treatment contributes to the distinction between intracranial hemorrhage and contrast staining.

Keywords Stroke $\cdot$ Endovascular treatment · Computed tomography $\cdot$ Spectral computed tomography $\cdot$ Intracerebral hemorrhage (ICH)

\section{Introduction}

Endovascular treatment (EVT) is the standard of care for acute ischemic stroke with large vessel occlusion in the anterior circulation $[1,2]$. Postprocedural computed tomography (CT) imaging can cause diagnostic difficulties, since hyperattenuation may represent either contrast extravasation

Marie Louise E. Bernsen

marie.1.e.bernsen@gmail.com

1 Department of Radiology, Rijnstate Hospital, Wagnerlaan 55, 6815 AD Arnhem, The Netherlands

2 Department of Neurology, Rijnstate Hospital, Arnhem, The Netherlands

3 Clinical Neurophysiology, Faculty of Science and Technology, University of Twente, Enschede, The Netherlands from the endovascular procedure, so called contrast staining, or intracranial hemorrhage (ICH) $[3,4]$. Hyperdense lesions on post-treatment CT are reported in up to $60 \%$ of the patients after EVT for large vessel occlusive stroke [3, $5,6]$ and symptomatic hemorrhagic transformation of the infarcted area is reported in $6 \%[2,7]$. The interpretation of hyperdense CT lesions may be relevant for decisions on antithrombotic management and general treatment decisions based on a patient's presumed prognosis [5]. Magnetic Resonance Imaging (MRI) does not offer a fixed solution in discriminating between iodine contrast and blood. It can lead to false positive hemorrhage due to the $\mathrm{T} 1$ time shortening effects from contrast administered during the endovascular procedure [8]. Furthermore, the availability of MRI in acute stroke imaging is limited [9].

Dual energy CT techniques have been proposed as a solution for discrimination of contrast enhancement and blood 
after EVT [10-13]. In these techniques, multiple x-ray photon energy spectra are used to distinguish materials with different attenuation properties at different energies, such as blood and iodine contrast agents [14]. The data obtained by these techniques can be used to reconstruct several imaging maps, such as virtual non-contrast images and effective atomic number maps. Roughly, the techniques can be divided in two groups: detector-based techniques and source-based techniques. Detector based techniques use dual-layered detector arrays to create dual-energy projection data from a conventional 120 or $140 \mathrm{kVp}$ scan [15]. This is a so-called retrospective technique and therefore no preselection is needed. Source based techniques are prospective techniques that need to be selected before the patient is scanned $[15,16]$. Examples of prospective techniques are dual-source scanning or single source twin beam.

Dual energy CT based on prospective techniques have been shown to modify the diagnosis of post-EVT ICH in around one third of the patients [10]. However, there are no reports on spectral detector based CT (SDCT) performance in this context [17].

We studied to what extent SDCT influences the diagnosis and classification of ICH in patients who received EVT for anterior circulation ischemic stroke.

\section{Methods}

\section{Design and patient selection}

We performed a retrospective analysis of CT data collected in the Rijnstate Hospital in Arnhem, The Netherlands. We included CT data from patients after EVT for ischemic stroke that underwent SDCT within 1 week after EVT in the period June 1, 2019 to February 1, 2021. We included CT data that were collected in the context of current care, because of neurological deterioration of a patient, and CT data that were collected during routine follow-up in study contexts (MRCLEAN MED [ISRCTN76741621], MR CLEAN NOiv [ISRCTN80619088], MR CLEAN Late studies [ISRCTN19922220]). If one patient received two spectral scans within one week, only the first scan was included in this study. All CT data were anonymized before analysis.

\section{Image acquisition and analysis}

Scans were obtained using the IQon spectral scanner (Philips Healthcare). This scanner consists of a dual layered detector to create dual energy data from a conventional $120 \mathrm{keV}$ scan. $\mathrm{CT}$ examinations were performed with a routine protocol with CTDIvol $16 \mathrm{~cm}$ of $31.8 \mathrm{mGy} \cdot \mathrm{cm}$. Routine thin slices $(0.9 \mathrm{~mm})$ and reconstructed axial and coronal 3-mm conventional polyenergetic $(120 \mathrm{kV}) \mathrm{CT}$ images, as well a virtual monoenergetic (or MonoE) $40 \mathrm{keV}$ were transferred to the Picture Archiving and Communication Systems (PACS) system. The conventional CT images were reconstructed using the iterative model reconstruction (IMR) technique (Philips Healthcare). Spectral postprocessing was performed using dedicated software (IntelliSpace Portal, Philips Healthcare). A virtual non-contrast scan (VNC), iodine removed map, and iodine map were generated. On the VNC scan, iodine pixels are identified and replaced by $\mathrm{HU}$ values meeting HU values in the absence of contrast enhancement. On the iodine removed map, these iodine pixels are replaced by black pixels. On the iodine map, pixel values represent the iodine concentration of the displayed tissue in $\mathrm{mg} / \mathrm{cc}$. Waterlike tissues are identified and suppressed. These image maps enhance visualization and distribution of iodine-enhanced tissue.

All CT images and maps were visually assessed by two experienced neuroradiologists. They analyzed the presence or absence of postprocedural hemorrhage on subsequent single energy CT and spectral CT, and classified the identified hemorrhages.

First, hyperattenuation was classified as either hemorrhage or contrast staining based on appearance on single energy CT. Second, the lesion was assessed on spectral $\mathrm{CT}$. The readers combined information of the VNC, iodine map, and iodine removed map to determine the nature of the lesion. Readers were blinded to clinical information and radiology reports. Disagreement was resolved through consensus during a joint reading session involving both readers.

We used the Heidelberg classification (Hemorrhagic infarction type 1 and type 2 [HI 1 and 2], parenchymal hematoma type 1 and type 2 [PH1 and 2], subarachnoidal hemorrhage [SAH], intraventricular hemorrhage [IVH], and subdural hemorrhage [SDH]) for diagnosing and grading of ICH [18]. For the sub classification of hemorrhages occurring remotely from infarcted tissue, the Safe Implementation of Thrombolysis in Stroke-Monitoring Study (SITSMOST) was followed ( $\mathrm{PHr} 1$ and $\mathrm{PHr} 2)$. The ICH grades were divided into 5 categories: no ICH, HI1 and/or HI2, SAH and/ or IVH, PH1 and/or PHr1, and PH2 and/or PHr2 (see Table 1 for definitions).

\section{Statistical analysis}

Data are presented in a descriptive way as median and interquartile range (IQR) or frequency and percentage (\%). Difference in proportions was tested with the Pearson's chi-squared test. Sensitivity, specificity, positive diagnostic value, negative diagnostic value, and overall accuracy of single $\mathrm{CT}$ diagnosis of ICH were calculated with $95 \%$ confidence intervals with SDCT as a reference. Unweighted and categorized quadratic weighted Cohen $\kappa$ values were used to assess the level of interreader agreement. All analyses were performed with SPSS 26 for Macintosh. 
Table 1 Classification of intracranial hemorrhage

\begin{tabular}{ll}
\hline Class & Definition \\
\hline HI1 & Scattered small petechiae along the margins of the infarct, no space-occupying effect \\
HI2 & Confluent petechiae within the infarcted area, no space-occupying effect \\
PH1 & Local, intra-ischemic, confluent hematoma in $\leq 30 \%$ of the infarcted area, no or mild space-occupying effect \\
PHr1 & Small to medium-sized hematoma located remote from the infarct(s), with mild space-occupying effect \\
PH2 & Local or intra-ischemic confluent hematoma $>30 \%$ of the infarcted area, with substantial space-occupying effect \\
PHr2 & Large confluent hematoma in an area remote from the actual infarct(s), with substantial space-occupying effect \\
IVH & Intraventricular hemorrhage \\
SAH & Subarachnoidal hemorrhage \\
\hline
\end{tabular}

Abbreviations: HII, hemorrhagic transformation type $1 ; H I 2$, hemorrhagic transformation type $2 ; P H 1$, parenchymal hemorrhage type $1 ; P H r 1$, parenchymal hemorrhage remote type $1 ; P H 2$, parenchymal hemorrhage type $2 ; P H r 2$, parenchymal hemorrhage remote type $2 ; I V H$, intraventricular hemorrhage; $S A H$, subarachnoidal hemorrhage

Table 2 Patient characteristics

\begin{tabular}{lll}
\hline Age, years (IQR) & 77 & $(66-83)$ \\
Male, n (\%) & 28 & $(44 \%)$ \\
NIHSS baseline, median (IQR) & 14 & $(7-19)$ \\
IVT, n (\%) & 36 & $(57 \%)$ \\
Transfer from other center, n (\%) & 30 & $(48 \%)$ \\
Occlusion segment, n (\%) & & \\
$\quad$ ICA-T & 3 & $(5 \%)$ \\
M1 & 32 & $(51 \%)$ \\
M2 & 26 & $(41 \%)$ \\
$\quad$ A2 & 1 & $(2 \%)$ \\
Basilar artery & 1 & $(2 \%)$ \\
Scan indication, n (\%) & & \\
$\quad$ Clinical deterioration & 35 & $(56 \%)$ \\
Study context & 27 & $(43 \%)$ \\
$\quad$ Inconclusive single energy CT & 1 & $(2 \%)$ \\
Time EVT - first SDCT scan, hours (IQR) & 21 & $(5-28)$ \\
\hline
\end{tabular}

Abbreviations: IQR, interquartile range; NIHSS, National Institute of Health Stroke Scale; ICA-T, internal carotid artery terminus segment; $\mathrm{M}$ (segment), middle cerebral artery; A(segment), anterior cerebral artery; $E V T$, endovascular treatment; SDCT, spectral detector CT

\section{Results}

Two-hundred-fifty-three endovascular procedures were performed in our center between June 1, 2019 and February 1,2020 . Postprocedural spectral CT within one week after the thrombectomy procedure was collected in 63 patients. Characteristics of this group are shown in Table 2. There was a slight female predominance and most patients had an occlusion in the proximal part of the middle cerebral artery. Most scans were collected because of clinical deterioration.

\section{Contrast staining vs. hemorrhage}

Contrast staining of the brain parenchym typically appeared as areas of hyperdensity, mostly confined to the grey matter (cortex and deep grey matter such as basal ganglia) (Fig. 1). Contrary to hemorrhagic lesions, no space occupying effect was seen. Contrast staining was misdiagnosed on single energy CT in four patients. Three of these patients were judged to have contrast staining on single energy CT, where on SDCT (small) additional hemorrhages were detected. One patient was judged to have a PH1 bleeding on single energy CT, where the hyperattenuation was judged to be contrast staining after assessment of SDCT data. In one patient classified as "no ICH" on single energy CT a small hemorrhagic lesion was detected by SDCT. This small hemorrhagic lesion was clearly visible on the MonoE images and VNC scan (Fig. 2).

\section{Diagnostic values}

Based on single energy CT, ICH was diagnosed in 32 patients. Based on spectral CT, this diagnosis changed in 8 patients $(P<0.001)$. In 4 patients, the diagnosis of $\mathrm{ICH}$ was rejected after assessment of SDCT and in 4 patients additional hemorrhage was detected. The grading of ICH changed in 3 patients. Distributions of intracranial hemorrhage, contrast staining only, intracranial hemorrhage grade, and intracranial hemorrhage on DECT are shown in Table 3.

Plain CT alone had a $88 \%$ (95\% CI 71-96\%) sensitivity, 87\% (95\% CI 70-96\%) specificity, 88\% (95\% CI 74-95\%) positive diagnostic value, $87 \%$ (95\% CI 73-94\%) negative diagnostic value, and $87 \%$ (95\% CI 77-94\%) overall accuracy for the dichotomous classification of any $\mathrm{ICH}$ vs no ICH. 
Fig. 1 Example of contrast staining. (a) On single energy $\mathrm{CT}$, an area of hyperattenuation is shown in the basal ganglia area (white arrow). There are no signs of space occupying effect, i.e. no compression on the right ventricle or midline shift. However, based on single energy CT additional hemorrhage cannot be ruled out completely. Eventually, this hyperattenuation was classified as contrast staining on single energy CT. (b) Virtual non-contrast (VNC) showing a small hyperdensity in the basal ganglia area, which may represent limited hemorrhagic transformation of the infarcted area $(\mathrm{H} 1)$. When combining the VNC with the iodine removal map (c) and iodine map (d) there is iodine removal in this area. This combination of single energy CT and all spectral reconstructions is typical for contrast staining with very limited hemorrhage
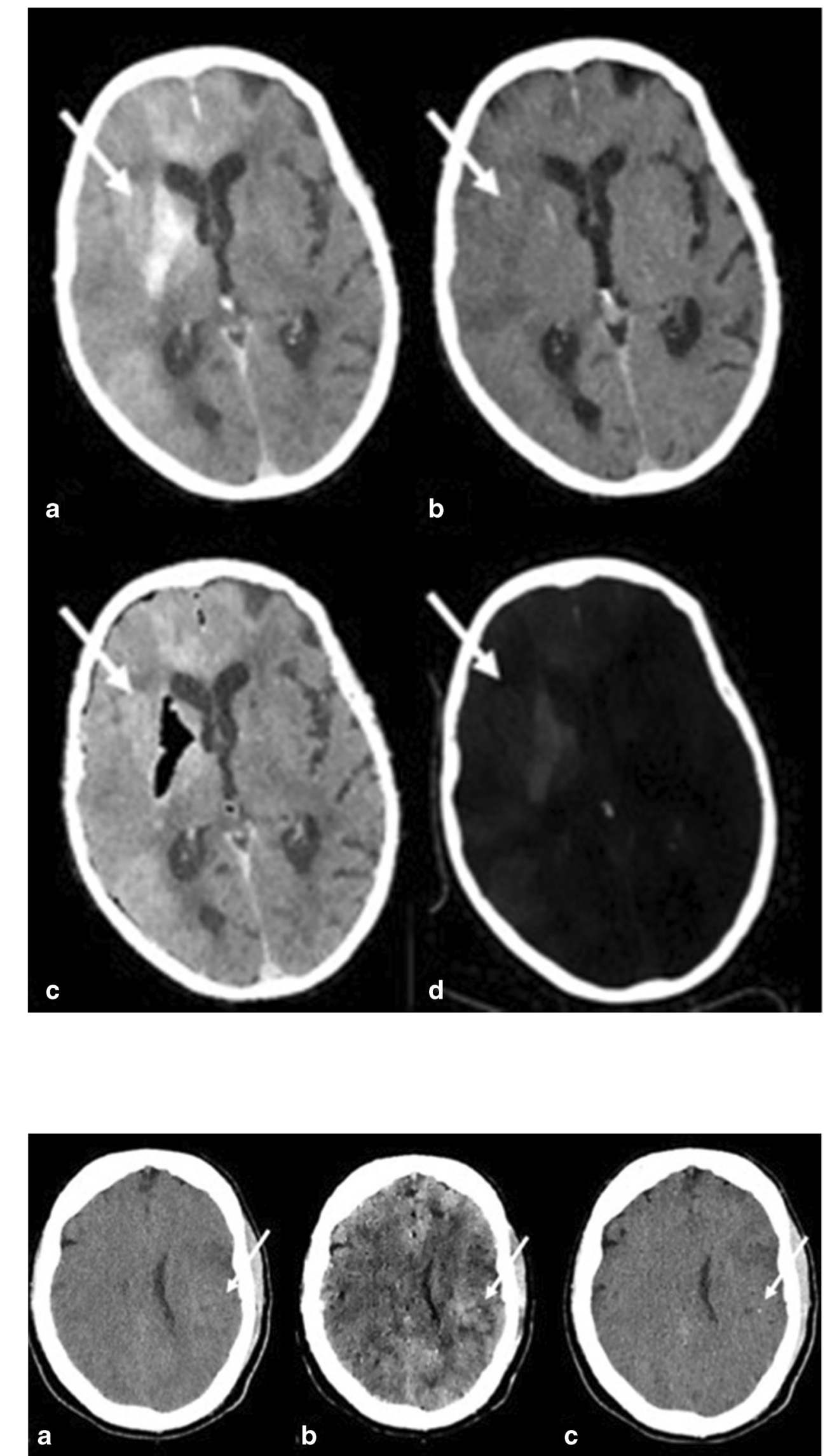

Fig. 2 Example of newly detected intracranial hemorrhage $(\mathrm{H} 1)$. On single energy $\mathrm{CT}$, the focal hyperattenuation is barely appreciated (a), however on MonoE (b) and virtual non-contrast images (c) a clear focal hyperattenuation is visible (white arrow). This patient was judged to have a petechial hemorrhage (H1) 
Table 3 ICH grade comparing single energy CT and SDCT after consensus

\begin{tabular}{llllll}
\hline Single energy CT, & $\mathrm{N}=63$ & $(\%)$ & SDCT, & $\mathrm{N}=63$ & \\
\hline No ICH & 31 & $(49 \%)$ & No ICH & 27 & $(43 \%)$ \\
& & & HI1/HI2 & 4 & $(6 \%)$ \\
HI1/HI2 & 5 & $(8 \%)$ & No ICH & 0 & $(0 \%)$ \\
& & & HI1/HI2 & 5 & $(8 \%)$ \\
SAH only & 13 & $(21 \%)$ & No ICH & 3 & $(5 \%)$ \\
& & & SAH & 10 & $(16 \%)$ \\
PH1/PHr1 & 9 & $(14 \%)$ & No ICH & 1 & $(2 \%)$ \\
& & & SAH & 1 & $(2 \%)$ \\
& & & H1/H2 & 1 & $(2 \%)$ \\
& & & PH1/PHr1 & 6 & $(10 \%)$ \\
PH2/PHr2 & 5 & $(8 \%)$ & No ICH & 0 & $(0 \%)$ \\
& & & PH 2/PHr 2 & 4 & $(6 \%)$ \\
& & & SAH & 1 & $(2 \%)$ \\
Total & 63 & & & 63 &
\end{tabular}

The HI1/HI2, $\mathrm{PH} 1 / \mathrm{PHr} 1$, and $\mathrm{PH} 2 / \mathrm{PHr} 2$ groups are with or without additional SAH and/or IVH. SAHs are with or without additional IVH

Abbreviations: $S D C T$, spectral detector CT; $I C H$, intracranial hemorrhage; HII, hemorrhagic transformation type $1 ; H I 2$, hemorrhagic transformation type 2; $\mathrm{PH}$, parenchymal hemorrhage type 1; PHrl, parenchymal hemorrhage remote type $1 ; P H 2$, parenchymal hemorrhage type 2; $\mathrm{PHr}$, parenchymal hemorrhage remote type 2; IVH, intraventricular hemorrhage; SAH, subarachnoidal hemorrhage

\section{Interreader agreement}

Interreader agreement of any ICH vs. no ICH was 0.84 (95\% CI 0.73-0.97) for single energy CT and 0.84 (95\% CI $0.51-0.86)$ for SDCT. Details of interreader reliability are presented in Table 4.

Table 4 Interreader agreement

\begin{tabular}{|c|c|c|}
\hline & $\begin{array}{l}\text { Single energy CT } \\
\kappa(95 \% \mathrm{CI})\end{array}$ & SDCT к $(95 \% \mathrm{CI})$ \\
\hline Any ICH vs no ICH & $0.84(0.73-0.97)$ & $0.84(0.51-0.86)$ \\
\hline ICH categorized unweighted & $0.64(0.50-0.78)$ & $0.64(0.50-0.79)$ \\
\hline ICH categorized, weighted & $0.83(0.72-0.94)$ & $0.77(0.63-0.91)$ \\
\hline
\end{tabular}

ICH was categorized as no ICH (1), SAH/IVH (1), H1/H2 (2), PH1/ PHr1(3) and $\mathrm{PH} 2 / \mathrm{PHr} 2(4)$.

The HI1/HI2, PH1/PHr1, and PH2 groups are with or without additional SAH and/or IVH. SAHs are with or without additional IVH

Abbreviations: $I C H$, intracranial hemorrhage; $S D C T$, spectra detector CT; $C I$, confidence interval

\section{Discussion}

SDCT changed the single energy CT diagnosis of postprocedural intracranial hemorrhage in 8 of 63 scans $(13 \%)$ in patients after EVT for ischemic stroke. These results are in line with other studies assessing the value of prospective dual energy CT techniques in post procedural imaging [10]. To the best of our knowledge, this is the first study to evaluate the additional value of SDCT after EVT for ischemic stroke.

\section{Dual energy CT techniques}

At present, several approaches to dual energy CT scanning are commercially available. Most used are scanners that consist of one or two tubes (such as rapid voltage switching and dual source CT) and scanners that use layered detectors (such as SDCT) [14]. Dual-source scanners consist of two source and detector combinations (Siemens AG), and use two simultaneously working X-ray tubes at different energy levels to create dual energy data. Single-source dual energy scanners with rapid kilovoltage switching (GE Healthcare) consist of a single tube and detector combination and use one tube that continuously alternates voltage during the scan. In TwinBeam dual energy CT (Siemens AG), spectral separation is also achieved at the level of the source. However, in contrary to fast peak kilovoltage switching, the beam is prefiltered and is split into high- and low-energy beams. The spectral detector dual energy CT scanner consists of one tube and detector. Contrary to the aforementioned techniques, the dual energy data is created on the detector side. The top layer absorbs the low energy photons, while the high energy photons pass through to the bottom layer [19].

With the spectral technique, there is no need for preselection in terms of alteration of scan parameters. Thus, work flow is unaffected. Also, there is no additional radiation dose and all spectral CT data can be analyzed retrospectively [20]. In addition, as opposed to prospective dual energy CT techniques [21], motion artifacts do not affect the spectral data reconstructions with SDCT, since spectral data are collected at the exact same time.

Spectral CT may also be superior to single energy CT in delineating the ischemic area after mechanical thrombectomy and has potential value in improving diagnostics outside the field of neuroradiology [22-24].

\section{Contrast staining and leakage}

Contrast staining represents areas of blood-brain barrier disruption causing increased microvascular permeability 
with extravasation of contrast agent into the extracellular space [25]. In the context of large vessel occlusion, contrast staining may result from ischemia or from direct damage to the vessel wall by thrombectomy devices [26]. Contrast agent leakage caused by damage of thrombectomy devices is mostly seen in the subarachnoid space [6]. Based on single energy CT, contrast staining can only be diagnosed if the attenuation value exceeds that expected for hemorrhage. However, only in the minority of cases with contrast enhancement such markedly elevated attenuation levels have been reported [27]. Furthermore, the leakage of iodine contrast agent may obscure hemorrhage on single energy CT (Fig. 3).

In a similar study performed by Almqvist et al., evaluating the influence of prospective dual energy CT using fast $\mathrm{Kv}$ switching on the diagnosis of post treatment $\mathrm{ICH}$, the proportion of patients diagnosed with $\mathrm{ICH}$ was significantly reduced from 41 to $27 \%$ [10]. In our study, in only $6 \%$ of the scans the diagnosis $\mathrm{ICH}$ was rejected based on spectral data.
With the growing amounts of patients receiving EVT, experience in contrast staining patterns increases for radiologist working in an endovascular treatment center. Based on this experience, many hyper-attenuation patterns were correctly classified as contrast staining based on single energy CT in our study, probably having contributed to the difference with the Almqvist analysis.

\section{Newly detected hemorrhage}

Conventional CT images are obtained using a heterochromatic $x$-ray spectrum. The created virtual monochromatic (MonoE) images display the attenuation values that would be expected if the patient could be imaged with an x-ray source of a fixed monochromatic energy value [13]. Low energy reconstructions show improved soft tissue contrast and higher energy images can reduce beam hardening artifacts arising from high attenuation materials such as bone (skull base) and metal. Several studies show that MonoE
Fig. 3 Example of leakage of contrast agent and blood in the subarachnoid space after endovascular procedure. On single energy CT, hyperdensity is seen in the subarachnoid space on the right side (a). On virtual noncontrast images (b) and iodine removal maps (c), part of this hyperdensity remains visible and on iodine map $(\mathbf{d})$, iodine is seen in the area. The combination of findings is characteristic for combined hemorrhage and contrast agent leakage

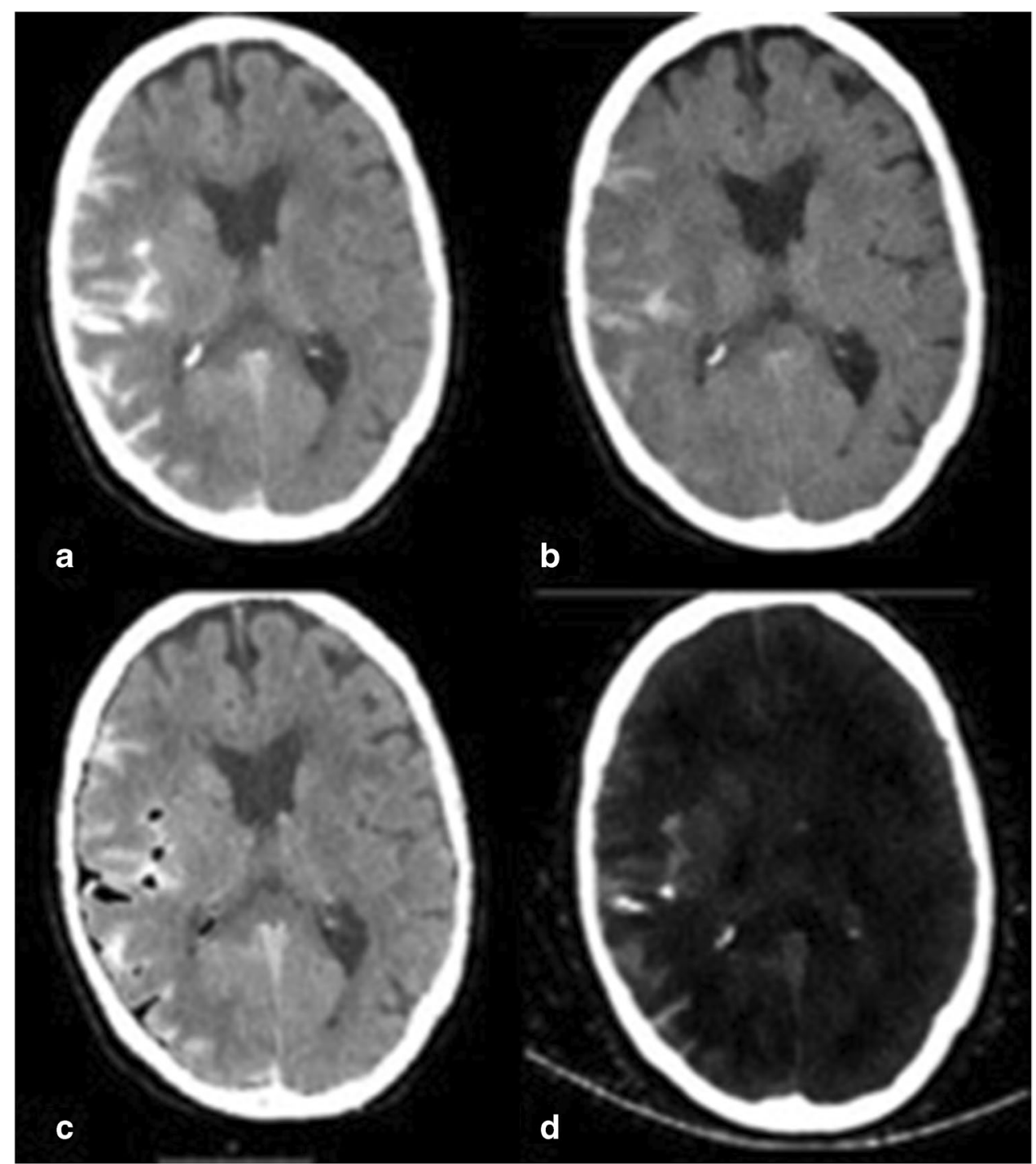


images with low energy levels of $40 \mathrm{keV}$ can help enhance lesion visualization and the detection rate of soft tissue lesions, for example small liver lesions, is increased [23, 28, 29].

We noticed higher conspicuity of hemorrhagic lesions on MonoE and VNC images.

\section{Limitations}

There are limitations to our study. This is a single center study and the number of patients is relatively small. In addition, clinical follow-up data are not included in our analysis.

This is not a study validating the spectral CT data. While phantom studies have shown the potential of SDCT for differentiating iodine from hemorrhage [30], without verification with MRI or neuropathology we cannot completely rule out any blood extravasation on SDCT images classified as no ICH. Dual energy CT material decomposition is usually successful in neuroradiology [21]. However, high density blood can sometimes be visible on iodine maps. Insufficient iodine suppression has been described, for example in leptomeningeal vessels, which led to inadequate diagnosis of hemorrhage in trauma settings [31]. Artifacts in CT, such as beam-hardening and metallic artifacts, can also affect dual energy analysis [21]. Finally, when using dual energy techniques outside of the field of neuroradiology, for example iodine quantitative assessments in oncology, it must be taken into account that several (patient) parameters can influence iodine concentrations [32]. To validate spectral CT in the brain, further study with comparison with susceptibility weighted MRI is needed, as it is unlikely that these MR sequences will be affected by the presence of iodine containing contrast agents [33].

In our study, interreader agreement was comparable for SDCT and single energy CT evaluation.

We conclude that SDCT is a simple and fast method that contributes to differentiation between intracerebral hemorrhage and contrast staining. It may be a valuable addition in hospitals who perform EVT to help the reporting radiologist in diagnosing postprocedural ICH.

Funding The authors did not receive support from any organization for the submitted work

Data availability Data cannot be made available, as no patient approval has been obtained for sharing coded data. However, syntax files and output of statistical analyses in SPSS will be made available on request.

\section{Declarations}

Conflict of interest The authors have no relevant financial or non-financial interests to disclose.
Ethical approval All procedures performed in the studies involving human participants were in accordance with the ethical standards of the institutional and/or national research committee and with the 1964 Helsinki Declaration and its later amendments or comparable ethical standards.

Informed consent No informed consent was obtained from the individual participants, due to the retrospective study design. Informed consent was given if the scan was made in study context.

\section{References}

1. Berkhemer O, Fransen P, Beumer D et al (2015) A randomized trial of intraarterial treatment for acute ischemic stroke. Engl J Med 372(1):11-20. https://doi.org/10.1056/NEJMoa1411587

2. Goyal M, Menon BK, Demchuk AM et al (2016) Endovascular thrombectomy after large-vessel ischaemic stroke: a meta-analysis of individual patient data from five randomised trials. Lancet 387(10029):1723-1731. https://doi.org/10.1016/S0140-6736(16) 00163-X

3. Nikoubashman O, Reich A, Gindullis M et al (2014) Clinical significance of post-interventional cerebral hyperdensities after endovascular mechanical thrombectomy in acute ischaemic stroke. Neuroradiology 56(1):41-50. https://doi.org/10.1007/ s00234-013-1303-1

4. Dekeyzer S, Nikoubashman O, Lutin B et al (2017) Distinction between contrast staining and hemorrhage after endovascular stroke treatment: one CT is not enough. J Neurointerv Surg 9:394-398. https://doi.org/10.1136/neurintsurg-2016-012290

5. Renú A, Amaro S, Laredo C et al (2015) Relevance of blood-brain barrier disruption after endovascular treatment of ischemic stroke dual-energy computed tomographic study. Stroke 46:673-679. https://doi.org/10.1161/STROKEAHA.114.008147

6. Parrilla G, García-Villalba B (2012) Espinosa De Rueda M, et al Hemorrhage/contrast staining areas after mechanical intra-arterial thrombectomy in acute ischemic stroke: imaging findings and clinical significance. AJNR Am J Neuroradiol 33(9):1791-1796. https://doi.org/10.3174/ajnr.A3044

7. Ivo GH, Jansen MJ, Mulder HL, Goldhoorn R-JB (2018) Endovascular treatment for acute ischaemic stroke in routine clinical practice: prospective, observational cohort study (MR CLEAN Registry). BMJ 360:k949. https://doi.org/10.1136/bmj.k949

8. Hergan K, Doringer W, Längle M, Oser W (1995) Effects of iodinated contrast agents in MR imaging. Eur J Radiol 21:11-17. https://doi.org/10.1016/0720-048x(95)00677-i

9. Yedavalli V (2017) Sammet S Contrast extravasation versus hemorrhage after thrombectomy in patients with acute stroke. J Neuroimaging 27(6):570-576. https://doi.org/10.1111/jon.12446

10. Almqvist H, Holmin S, Mazya MV (2019) Dual energy CT after stroke thrombectomy alters assessment of hemorrhagic complications. Neurology 93(11):e1068-e1075. https://doi.org/10.1212/ WNL.0000000000008093

11. Tijssen MPM, Hofman PAM, Stadler AAR et al (2014) The role of dual energy CT in differentiating between brain haemorrhage and contrast medium after mechanical revascularisation in acute ischaemic stroke. Eur Radiol 24(4):834-840. https://doi.org/10. 1007/s00330-013-3073-x

12. Watanabe Y, Tsukabe A, Kunitomi Y et al (2014) Dual-energy CT for detection of contrast enhancement or leakage within highdensity haematomas in patients with intracranial haemorrhage. Neuroradiology 56(4):291-295. https://doi.org/10.1007/s00234014-1333-3.13 
13. Dual-Energy CT in Emergency Neuroimaging: Added Value and Novel Applications 1. https://doi.org/10.1148/rg.2016160069

14. Johnson TRC (2012) (2012) Dual-energy CT: general principles. AJR Am J Roentgenol 199(5 Suppl):S3-8. https://doi.org/10.2214/ AJR.12.9116

15. Jacobsen MC, Schellingerhout D, Wood CA et al (2018) (2018) intermanufacturer comparison of dual-energy $\mathrm{cT}$ iodine quantification and monochromatic attenuation: a phantom study. Radiology 287(1):224-234. https://doi.org/10.1148/radiol.2017170896

16. Patino M, Prochowski A, Agrawal MD et al (2016) Material separation using dual-energy CT: current and emerging applications. Radiographics 36(4):1087-1105. https://doi.org/10.1148/rg.20161 50220

17. Choi Y, Shin NY, Jang J et al (2020) Dual-energy CT for differentiating acute intracranial hemorrhage from contrast staining or calcification: a meta-analysis. Neuroradiology 62(12):1617-1626. https://doi.org/10.1007/s00234-020-02486-w

18. Von Kummer R, Broderick JP, Campbell BCV et al (2015) The heidelberg bleeding classification: classification of bleeding events after ischemic stroke and reperfusion therapy. Stroke 46(10):2981-2986. https://doi.org/10.1161/STROKEAHA.115. 010049

19. Forghani R, De Man B, Gupta R (2017) Dual-energy computed tomography physical principles, approaches to scanning, usage, and implementation: part 1. Neuroimaging Clin N Am 27(3):371384. https://doi.org/10.1016/j.nic.2017.03.002

20. Rassouli N, Etesami M, Dhanantwari A, Rajiah P (2017) Detectorbased spectral CT with a novel dual-layer technology: principles and applications. Insights Imaging 8(6):589-598. https://doi.org/ 10.1007/s13244-017-0571-4

21. Dinkel J, Khalilzadeh O, Phan CM et al (2015) Technical limitations of dual-energy CT in neuroradiology: 30-month institutional experience and review of literature. J Neurointerv Surg 7(8):596602. https://doi.org/10.1136/neurintsurg-2014-011241

22. Riederer I, Fingerle AA, Baum T et al (2018) Acute infarction after mechanical thrombectomy is better delineable in virtual non-contrast compared to conventional images using a duallayer spectral CT. Sci Rep 8(1):9329. https://doi.org/10.1038/ s41598-018-27437-7

23. Lennartz S, Große Hokamp N, Abdullayev N et al (2019) Diagnostic value of spectral reconstructions in detecting incidental skeletal muscle metastases in CT staging examinations. Cancer Imaging 19(1):50. https://doi.org/10.1186/s40644-019-0235-3

24. Lv P, Lin XZ, Chen K, Gao J (2012) Spectral CT in patients with small HCC: Investigation of image quality and diagnostic accuracy. Eur Radiol 22(10):2117-2124. https://doi.org/10.1007/ s00330-012-2485-3
25. Hamann GF, Okada Y, Del Zoppo GJ (1996) Hemorrhagic transformation and microvascular integrity during focal cerebral ischemia/reperfusion. J Cereb Blood Flow Metab 16(6):13731378. https://doi.org/10.1097/00004647-199611000-00036

26. Peschillo S, Diana F, Berge J, Missori P (2016) A comparison of acute vascular damage caused by ADAPT versus a stent retriever device after thrombectomy in acute ischemic stroke: a histological and ultrastructural study in an animal model. J Neurointerv Surg. 2017 Aug;9(8):743-749.https://doi.org/10.1136/neuri ntsurg-2016-012533

27. Gupta R, Phan CM, Leidecker C et al (2010) Evaluation of dualenergy CT for differentiating intracerebral hemorrhage from iodinated contrast material staining. Radiology 257(1):205-211. https://doi.org/10.1148/radiol.10091806

28. Gao QZ, Wang ZW, Qi YF, Jin ZY (2018) Monoenergetic reconstructions using detector-based spectral CT for imaging of small lesions in the rabbit VX2 liver cancer models. Zhongguo Yi Xue Ke Xue Yuan Xue Bao 40(5):651-655. https://doi.org/10.3881/j. issn.1000-503X.10257

29. Matsumoto K, Jinzaki M, Tanami Y et al (2011) Virtual monochromatic spectral imaging with fast kilovoltage switching: improved image quality as compared with that obtained with conventional 120-kVp CT. Radiology 259(1):257-262. https:// doi.org/10.1148/radiol.11100978

30. Van Hedent S, Große Hokamp N, Laukamp KR et al (2018) Differentiation of hemorrhage from iodine using spectral detector CT: a phantom study. AJNR Am J Neuroradiol 39(12):2205-2210. https://doi.org/10.3174/ajnr.A5872

31. Cho SB, Baek HJ, Ryu KH et al (2017) Initial clinical experience with dual-layer detector spectral CT in patients with acute intracerebral haemorrhage: a single-centre pilot study. PLoS ONE 12(11):e0186024. https://doi.org/10.1371/journal.pone.0186024

32. Corrias G, Sawan P, Mahmood U, Zheng J, Capanu M, Salvatore M, Spinato G, Saba L, Mannelli L (2019) Dual energy computed tomography analysis in cancer patients: what factors affect iodine concentration in contrast enhanced studies? Eur J Radiol 120:108698. https://doi.org/10.1016/j.ejrad.2019.108698

33. Nikoubashman XO, Jablawi XF, Dekeyzer XS et al (2016) MRI appearance of intracerebral iodinated contrast agents: is it possible to distinguish extravasated contrast agent from hemorrhage? AJNR Am J Neuroradiol 37(8):1418-1421. https://doi.org/10. 3174/ajnr.A4755

Publisher's note Springer Nature remains neutral with regard to jurisdictional claims in published maps and institutional affiliations. 